\section{Tetranucleotide repeat polymorphism at the HPRT locus}

\author{
C.M.Hearne and J.A.Todd * \\ Nuffield Department of Surgery, John Radcliffe Hospital, \\ Headington, Oxford OX3 9DU, UK
}

Source/Description: A polymorphic (TCTA) 13 repeat is located at base 22928 of the human HPRT locus (1). The polymorphism can be typed using the polymerase chain reaction (PCR) and gel electrophoresis as described previously (2). The predicted length of the amplified sequence is $159 \mathrm{bp}$.

\section{Primer Sequences: \\ Forward 5'TCTCTATTTCCATCTCTGTCTCC Reverse 5'TCACCCCTGTCTATGGTCTCG}

Frequency: Estimated from 48 chromosomes of unrelated individuals, $\mathrm{PIC}=0.70$.
Allele (bp)
Frequency
D1 163
0.167
D2 159
0.396
Allele (bp) Frequency
D3 $155 \quad 0.292$
D4 $151 \quad 0.104$

Mendelian Inheritance: Co-dominant X-linked inheritance was observed in six two-generation families.

Chromosomal Localisation: The HPRT locus is located on chromosome Xq26 (3).

Other Comments: PCR was performed with $250 \mathrm{ng}$ of genomic DNA and $125 \mathrm{ng}$ of each oligonucleotide primer in a $25 \mu \mathrm{l}$ volume as described previously (2).

References: 1) Edwards,A. et al. (1990) Genomics 6, 593-608. 2) Love,J.M. et al. (1990) Nucl. Acids Res. 18, 4123-4130. 3) Williamson, R. et al. (1990) Cytogenet. Cell Genet. 55, 475-778.

\section{Trinucleotide repeat polymorphism at the CRYG1 locus}

C.M.Hearne and J.A.Todd *

Nuffield Department of Surgery, John Radcliffe Hospital, Headington, Oxford OX3 9DU, UK

Source/Description: A polymorphic (AAC) $)_{9}$ repeat is located at base 3021 of the human gamma B crystallin (HGM symbol CRYG1, EMBL Accession M19354). The polymorphism can be typed using the polymerase chain reaction (PCR) and gel electrophoresis as described previously (1). The predicted length of the amplified sequence was $182 \mathrm{bp}$.

Primer Sequences:

Forward 5'GCCTGGCGACAGAGTGAGAC

Reverse 5'AAGTGAGCTAATAGGTACTGACC

Frequency: Estimated from 48 chromosomes of unrelated individuals, $\mathrm{PIC}=0.55$.

$\begin{array}{ll}\text { Allele (bp) } & \text { Frequency } \\ \text { E1 } 191 & 0.266 \\ \text { E2 } 188 & 0.500 \\ \text { E3 } 182 & 0.234\end{array}$

Mendelian Inheritance: Co-dominant segregation was observed in six two-generation informative families.

Chromosomal Localisation: The CRYGl is located on chromosome 2q33-q35 (2).

Other Comments: PCR was performed on $250 \mathrm{ng}$ of genomic DNA using $125 \mathrm{ng}$ each oligonucleotide primer in a volume of $25 \mu \mathrm{l}$, and the samples processed as described previously (1).

References: 1) Love,J.M. et al. (1990) Nucl. Acids Res. 18, 4123-4130. 2) Williamson, R. et al. (1990) Cytogenet. Cell Genet. 55, 475-778. 